\title{
Balancing related methods for minimal realization of periodic systems
}

\author{
A. Varga ${ }^{1}$ \\ German Aerospace Center, DLR-Oberpfaffenhofen, Institute of Robotics and System Dynamics, D-82234 Wessling, Germany
}

Received 31 August 1998; received in revised form 20 November 1998

\begin{abstract}
We propose balancing related numerically reliable methods to compute minimal realizations of linear periodic systems with time-varying dimensions. The first method belongs to the family of square-root methods with guaranteed enhanced computational accuracy and can be used to compute balanced minimal order realizations. An alternative balancing-free square-root method has the advantage of a potentially better numerical accuracy in case of poorly scaled original systems. The key numerical computation in both methods is the solution of nonnegative periodic Lyapunov equations directly for the Cholesky factors of the solutions. For this purpose, a numerically reliable computational algorithm is proposed to solve nonnegative periodic Lyapunov equations with time-varying dimensions. (c) 1999 Elsevier Science B.V. All rights reserved.
\end{abstract}

Keywords: Numerical methods; Linear time-varying systems; Periodic systems; Balancing transformation; Minimal realization

\section{Introduction}

In the last few years there has been a constantly increasing interest to develope numerical algorithms for the analysis and design of linear periodic discrete-time control systems with constant state-, input- and output-vector dimensions $[2,8,11,15,16]$. Areas where significant theoretical results have been achieved for periodic systems with time-varying state dimensions are the solution of the minimal realization problem $[4,5]$ and robust pole assignment [9].

In this paper we develop balancing related numerical methods to compute minimal realizations of linear periodic systems with time-varying dimensions. The first method can be used to compute balanced minimal realizations and belongs to the family of square-root methods with guaranteed enhanced computational accuracy. The matrices of the minimal realization are computed using appropriate truncation matrices determined exclusively using the Cholesky factors of the periodic reachability and observability Gramians.

An alternative balancing-free square-root method for minimal realization has the advantage of a potentially better numerical accuracy in case of poorly scaled original nonminimal systems. By avoiding the use of possibly ill-conditioned balancing based truncation formulas and by using instead well-conditioned

${ }^{1}$ E-mail: andras.varga@dlr.de. 
transformations, the accuracy of computations can be often significantly improved. The second method extends the balancing-free square-root approach for standard systems [14] to periodic systems.

The key computation in the proposed computational approaches is the solution of nonnegative periodic Lyapunov equations directly for the Cholesky factors of the Gramians. For this purpose, a numerically reliable computational algorithm is proposed to solve nonnegative periodic Lyapunov equations with time-varying dimensions. The proposed algorithm is an extension of a method proposed by the author for constant dimensions $[15]$.

\section{Preliminaries}

Consider the linear discrete-time $K$-periodic system

$$
\begin{aligned}
& x_{k+1}=A_{k} x_{k}+B_{k} u_{k}, \\
& y_{k}=C_{k} x_{k},
\end{aligned}
$$

where the matrices $A_{k} \in \mathbb{R}^{n_{k+1} \times n_{k}}, B_{k} \in \mathbb{R}^{n_{k+1} \times m_{k}}, C_{k} \in \mathbb{R}^{p_{k} \times n_{k}}$ and the integers $n_{k}, m_{k}$, $p_{k}$ are periodic with period $K \geqslant 1$. The transition matrix of the system (1) is defined by the $n_{j} \times n_{i}$ matrix $\Phi_{A}(j, i)=A_{j-1} A_{j-2} \cdots A_{i}$, where $\Phi_{A}(i, i):=I_{n_{i}}$. The state transition matrix over one period $\Phi_{A}(j+K, j) \in \mathbb{R}^{n_{j} \times n_{j}}$ is called the monodromy matrix of system (1) at time $j$ and its eigenvalues are called characteristic multipliers at time $j$. Note that $\Lambda\left(\Phi_{A}(j+K, j)\right)$ has always at least $n_{j}-\underline{n}$ zero elements, where $\underline{n}:=\min _{k}\left\{n_{k}\right\}$. The rest of $\underline{n}$ eigenvalues are independent of time $j$ and form the core characteristic multipliers [6]. The periodic system (1) is asymptotically stable if all characteristic multipliers belong to the open unit disk. For the definitions of reachability, observability and minimality of periodic systems we use the corresponding notions from [5] for general time-varying systems.

Definition 1. The periodic system (1) is reachable at time $k$ if

$$
\operatorname{rank} G_{k}=n_{k} \text {, }
$$

where $G_{k}$ is the infinite columns matrix

$$
G_{k}=\left[B_{k-1} A_{k-1} B_{k-2} \cdots \Phi_{A}(k, i+1) B_{i} \cdots\right] .
$$

The periodic system (1) is completely reachable if (2) holds for all $k$.

Definition 2. The periodic system (1) is observable at time $k$ if

$$
\operatorname{rank} F_{k}=n_{k},
$$

where $F_{k}$ is the infinite rows matrix

$$
F_{k}=\left[\begin{array}{c}
C_{k} \\
C_{k+1} A_{k} \\
\vdots \\
C_{i} \Phi_{A}(i, k) \\
\vdots
\end{array}\right] .
$$

The periodic system (1) is completely observable if (4) holds for all $k$.

Definition 3. The periodic system (1) is minimal if it is completely reachable and completely observable. 
For an asymptotically stable periodic system, the $n_{k} \times n_{k}$ reachability Gramian at time $k$ is defined as

$$
P_{k}:=\sum_{i=-\infty}^{k-1} \Phi_{A}(k, i+1) B_{i} B_{i}^{\mathrm{T}} \Phi_{A}(k, i+1)^{\mathrm{T}}=G_{k} G_{k}^{\mathrm{T}} \geqslant 0,
$$

where $G_{k}$ is defined in Eq. (3). Similarly, the $n_{k} \times n_{k}$ observability Gramian at time $k$ is defined as

$$
Q_{k}=\sum_{i=k}^{\infty} \Phi_{A}(i, k)^{\mathrm{T}} C_{i}^{\mathrm{T}} C_{i} \Phi_{A}(i, k)=F_{k}^{\mathrm{T}} F_{k} \geqslant 0,
$$

with $F_{k}$ defined by Eq. (5). Note that both Gramians are $K$-periodic matrices. Using the definitions of reachability and observability we have the following results.

Proposition 1. The periodic system (1) is reachable at time $k$ if $P_{k}>0$ and is completely reachable if $P_{k}>0$ for $k=0, \ldots, K-1$.

Proposition 2. The periodic system (1) is observable at time $k$ if $Q_{k}>0$ and is completely observable if $Q_{k}>0$ for $k=0, \ldots, K-1$.

Notation. For a $K$-periodic matrix $X_{k}$ we use alternatively the script notation

$$
\mathscr{X}:=\operatorname{diag}\left(X_{0}, X_{1}, \ldots, X_{K-1}\right),
$$

which associates the block-diagonal matrix $\mathscr{X}$ to the cyclic matrix sequence $X_{k}, k=0, \ldots, K-1$. This notation is consistent with the standard matrix operations as for instance addition, multiplication, inversion as well as with several standard matrix decompositions (Cholesky, SVD). We denote with $\sigma \mathscr{X}$ the $K$-cyclic shift

$$
\sigma \mathscr{X}=\operatorname{diag}\left(X_{1}, \ldots, X_{K-1}, X_{0}\right)
$$

of the cyclic sequence $X_{k}, k=0, \ldots, K-1$. By using the script notation, the periodic system (1) will be alternatively denoted by the quadruple $(\mathscr{A}, \mathscr{B}, \mathscr{C})$.

\section{Square-root minimal realization}

For an asymptotically stable periodic system the two Gramians are nonnegative definite and satisfy nonnegative (or positive) discrete periodic Lyapunov equations (PDPLEs) as follows: the reachability Gramian $\mathscr{P}$ satisfies the forward-time PDPLE

$$
\sigma \mathscr{P}=\mathscr{A} \mathscr{P} \mathscr{A}^{\mathrm{T}}+\mathscr{B} \mathscr{B}^{\mathrm{T}},
$$

while the observability Gramian 2 satisfies the reverse-time PDPLE

$$
\mathscr{Q}=\mathscr{A}^{\mathrm{T}} \sigma \mathscr{Q} \mathscr{A}+\mathscr{C}^{\mathrm{T}} \mathscr{C} .
$$

Let $T_{k} \in \mathbb{R}^{n_{k} \times n_{k}}$ be a $K$-periodic invertible matrix. Two periodic systems $(\mathscr{A}, \mathscr{B}, \mathscr{C})$ and $(\tilde{\mathscr{A}}, \widetilde{\mathscr{B}}, \widetilde{\mathscr{C}})$ related by the transformation

$$
(\widetilde{\mathscr{A}}, \widetilde{\mathscr{B}}, \widetilde{\mathscr{C}})=\left(\sigma \mathscr{T}^{-1} \mathscr{A} \mathscr{T}, \sigma \mathscr{T}^{-1} \mathscr{B}, \mathscr{C} \mathscr{T}\right)
$$

are called Lyapunov-similar and Eq. (8) is called a Lyapunov similarity transformation. The Gramians $\widetilde{\mathscr{P}}$ and $\widetilde{\mathscr{Q}}$ of the transformed system $(\widetilde{\mathscr{A}}, \widetilde{\mathscr{B}}, \widetilde{\mathscr{C}})$ satisfy

$$
\widetilde{\mathscr{P}}=\mathscr{T}^{-1} \mathscr{P} \mathscr{T}^{-T}, \quad \widetilde{\mathcal{Q}}=\mathscr{T}^{\mathrm{T}} \mathscr{Q} \mathscr{T} .
$$

For a completely reachable and completely observable (i.e., minimal) periodic system, $\mathscr{T}$ can be determined such that the transformed Gramians are equal and diagonal and thus the transformed periodic system is 
balanced [15]. The diagonal elements of the balanced Gramians are called the Hankel singular values and are the positive square-roots of the eigenvalues of the product $\mathscr{P} \mathscr{Q}$. The maximum of them defines the Hankel-norm of the given periodic system [1].

Let $\mathscr{P}=\mathscr{S}^{\mathrm{T}} \mathscr{S}$ and $\mathscr{Q}=\mathscr{R}^{\mathrm{T}} \mathscr{R}$ be in Cholesky factorized forms. For a minimal system, in analogy with the standard case [13], we can use the singular value decomposition

$$
\mathscr{R} \mathscr{S}^{\mathrm{T}}=\mathscr{U} \Sigma \mathscr{V}^{\mathrm{T}}
$$

to compute the balancing transformation matrix $\mathscr{T}$ and its inverse $\mathscr{T}^{-1}$ as

$$
\mathscr{T}=\mathscr{S}^{\mathrm{T}} \mathscr{V} \Sigma^{-1 / 2}, \quad \mathscr{T}^{-1}=\Sigma^{-1 / 2} \mathscr{U}^{\mathrm{T}} \mathscr{R} .
$$

For an asymptotically stable non-minimal periodic system $(\mathscr{A}, \mathscr{B}, \mathscr{C})$, the balancing transformation is not defined because the system is not completely reachable and/or not completely observable, and thus, from Proposition 1 or $2 \Sigma$ in Eq. (9) is not invertible. We will show how it is possible to determine a realization of the given periodic system which is minimal, i.e., completely reachable and completely observable, and moreover balanced. The main result (Theorem 1) of this section can be seen as an extension to the periodic case of a similar result in [14] for standard systems.

Let us write the singular value decomposition (9) at each time instant $k$ in the partitioned form

$$
R_{k} S_{k}^{\mathrm{T}}=\left[U_{k, 1} U_{k, 2}\right]\left[\begin{array}{cc}
\widetilde{\Sigma}_{k} & 0 \\
0 & 0
\end{array}\right]\left[V_{k, 1} V_{k, 2}\right]^{\mathrm{T}},
$$

where $\widetilde{\Sigma}_{k} \in \mathbb{R}^{r_{k} \times r_{k}}, U_{k, 1} \in \mathbb{R}^{n_{k} \times r_{k}}, V_{k, 1} \in \mathbb{R}^{n_{k} \times r_{k}}$ and $\widetilde{\Sigma}_{k}>0$. From the above decomposition define, with $\widetilde{\Sigma}=\operatorname{diag}\left(\widetilde{\Sigma}_{0}, \ldots, \widetilde{\Sigma}_{K-1}\right)$, the truncation matrices

$$
\mathscr{L}=\widetilde{\Sigma}^{-1 / 2} \mathscr{U}_{1}^{\mathrm{T}} \mathscr{R}, \quad \mathscr{T}=\mathscr{S}^{\mathrm{T}} \mathscr{V}_{1} \widetilde{\Sigma}^{-1 / 2},
$$

which are used to determine the reduced system matrices

$$
\widehat{\mathscr{A}}=\sigma \mathscr{L} \mathscr{A} \mathscr{T}, \quad \widehat{\mathscr{B}}=\sigma \mathscr{L} \mathscr{B}, \quad \widehat{\mathscr{C}}=\mathscr{C} \mathscr{T} .
$$

The following theorem is the main theoretical result of the paper.

Theorem 1. The periodic system $(\widehat{\mathscr{A}}, \widehat{\mathscr{B}}, \widehat{\mathscr{C}})$ defined in Eq. (12) is a balanced minimal realization of the system $(\mathscr{A}, \mathscr{B}, \mathscr{C})$.

Proof. The proof is in two steps. First we prove that $(\widehat{\mathscr{A}}, \widehat{\mathscr{B}}, \widehat{\mathscr{C}})$ is a minimal balanced realization and then we prove that it realizes the same input-output operator as $(\mathscr{A}, \mathscr{B}, \mathscr{C})$.

Let substitute $\mathscr{P}=\mathscr{S}^{\mathrm{T}} \mathscr{S}$ in Eq. (6) and premultiply it by $\sigma \mathscr{L}$ and postmultiply it by $\sigma \mathscr{L}^{\mathrm{T}}$ to obtain

$$
\sigma\left(\mathscr{L} \mathscr{S}^{\mathrm{T}} \mathscr{S} \mathscr{L}^{\mathrm{T}}\right)=\sigma \mathscr{L} \mathscr{A} \mathscr{S}^{\mathrm{T}} \mathscr{S} \mathscr{A}^{\mathrm{T}} \sigma \mathscr{L}^{\mathrm{T}}+\sigma \mathscr{L} \mathscr{B} \mathscr{B}^{\mathrm{T}} \sigma \mathscr{L}^{\mathrm{T}}
$$

It can be readily verified that

$$
\sigma\left(\mathscr{L} \mathscr{S}^{\mathrm{T}} \mathscr{S} \mathscr{L}^{\mathrm{T}}\right)=\sigma \widetilde{\Sigma} .
$$

From Eq. (9) we also have

$$
\mathscr{R} \mathscr{S}^{\mathrm{T}} \mathscr{V}_{2}=0 .
$$


Now, we replace $\mathscr{Q}=\mathscr{R}^{\mathrm{T}} \mathscr{R}$ in Eq. (7) and premultiply this equation by $\mathscr{V}_{2}^{\mathrm{T}} \mathscr{S}$ and postmultiply it by $\mathscr{S}^{\mathrm{T}} \mathscr{V}_{2}$, to obtain, using also Eq. (15),

$$
\mathscr{V}_{2}^{\mathrm{T}} \mathscr{S} \mathscr{A}^{\mathrm{T}} \sigma \mathscr{R}^{\mathrm{T}} \sigma \mathscr{R} \mathscr{A} \mathscr{S}^{\mathrm{T}} \mathscr{V}_{2}+\mathscr{V}_{2}^{\mathrm{T}} \mathscr{S} \mathscr{C}^{\mathrm{T}} \mathscr{C} \mathscr{S}^{\mathrm{T}} \mathscr{V}_{2}=0
$$

It follows immediately that $\mathscr{C} \mathscr{S}^{\mathrm{T}} \mathscr{V}_{2}=0$ and also

$$
\sigma \mathscr{R} \mathscr{A} \mathscr{S}^{\mathrm{T}} \mathscr{V}_{2}=0 .
$$

Using the identity $\mathscr{V}_{1} \mathscr{V}_{1}^{\mathrm{T}}+\mathscr{V}_{2} \mathscr{V}_{2}^{\mathrm{T}}=I$ and Eq. (16), as well as the expressions for $\mathscr{L}$ and $\mathscr{T}$, the first term on the right-hand side of Eq. (13) becomes successively

$$
\begin{aligned}
& \sigma \mathscr{L} \mathscr{A} \mathscr{S}^{\mathrm{T}} \mathscr{S} \mathscr{A}^{\mathrm{T}} \sigma \mathscr{L}^{\mathrm{T}}=\sigma \mathscr{L} \mathscr{A} \mathscr{S}^{\mathrm{T}} \mathscr{V}_{1} \mathscr{V}_{1}^{\mathrm{T}} \mathscr{S} \mathscr{A}^{\mathrm{T}} \sigma \mathscr{L}^{\mathrm{T}}+\sigma \widetilde{\Sigma}^{-1 / 2} \sigma \mathscr{U}_{1}^{\mathrm{T}} \sigma \mathscr{R} \mathscr{A} \mathscr{S}^{\mathrm{T}} \mathscr{V}_{2} \mathscr{V}_{2}^{\mathrm{T}} \mathscr{S} \mathscr{A}^{\mathrm{T}} \sigma \mathscr{R}^{\mathrm{T}} \sigma \mathscr{U}_{1} \sigma \widetilde{\Sigma}^{-1 / 2} \\
& =\sigma \mathscr{L} \mathscr{A} \mathscr{T} \widetilde{\Sigma} \mathscr{T}^{\mathrm{T}} \mathscr{A}^{\mathrm{T}} \sigma \mathscr{L}^{\mathrm{T}} \text {. }
\end{aligned}
$$

Now, by using the definitions of $\widehat{\mathscr{A}}$ and $\widehat{\mathscr{B}}$ in Eq. (12), and taking into account Eqs. (14) and (17), Eq. (13) becomes

$$
\sigma \widetilde{\Sigma}=\widehat{\mathscr{A}} \widetilde{\Sigma} \widehat{\mathscr{A}}^{\mathrm{T}}+\widehat{\mathscr{B}}^{\mathrm{T}} \mathrm{B} .
$$

In a similar way we can show that

$$
\widetilde{\Sigma}=\widehat{\mathscr{A}}^{\mathrm{T}} \sigma \widetilde{\Sigma} \widehat{\mathscr{A}}+\widehat{\mathscr{C}}^{\mathrm{T}} \widehat{\mathscr{C}}
$$

and thus $(\widehat{\mathscr{A}}, \widehat{\mathscr{B}}, \widehat{\mathscr{C}})$ is balanced. Since $\widetilde{\Sigma}>0$, from Eqs. (18) and (19) it follows that $(\widehat{\mathscr{A}}, \widehat{\mathscr{B}}, \widehat{\mathscr{C}})$ is completely reachable and completely observable, and thus also minimal [5]. Moreover, the minimal system is also asymptotically stable.

To complete the proof it remains to show that the two realizations $(\widehat{\mathscr{A}}, \widehat{\mathscr{B}}, \widehat{\mathscr{C}})$ and $(\mathscr{A}, \mathscr{B}, \mathscr{C})$ achieve the same input-output operator. To this end, we define the error system $\left(\mathscr{A}_{e}, \mathscr{B}_{e}, \mathscr{C}_{e}\right)$ by

$$
A_{k, e}=\left[\begin{array}{cc}
A_{k} & 0 \\
0 & \widehat{A}_{k}
\end{array}\right], \quad B_{k, e}=\left[\begin{array}{c}
B_{k} \\
\widehat{B}_{k}
\end{array}\right], \quad C_{k, e}=\left[C_{k}-\widehat{C}_{k}\right] .
$$

The reachability Gramian $\mathscr{P}_{e}$ of the error system satisfies the periodic Lyapunov equation

$$
\sigma \mathscr{P}_{e}=\mathscr{A}_{e} \mathscr{P}_{e} \mathscr{A}_{e}^{\mathrm{T}}+\mathscr{B}_{e} \mathscr{B}_{e}^{\mathrm{T}}
$$

If we partition $P_{k, e}$ in the form

$$
P_{k, e}=\left[\begin{array}{ll}
P_{k, 11} & P_{k, 12} \\
P_{k, 12}^{\mathrm{T}} & P_{k, 22}
\end{array}\right]
$$

then we obtain immediately that $\mathscr{P}_{11}=\mathscr{S}^{\mathrm{T}} \mathscr{S}, \mathscr{P}_{22}=\widetilde{\Sigma}$, and $\mathscr{P}_{12}$ satisfies the periodic Sylvester equation

$$
\sigma \mathscr{P}_{12}=\mathscr{A} \mathscr{P}_{12} \widehat{\mathscr{A}}^{\mathrm{T}}+\mathscr{B} \widehat{\mathscr{B}}^{\mathrm{T}}
$$

This equation has a unique solution guaranteed by the asymptotic stability of both the non-minimal and minimal realizations.

After substituting $\widehat{\mathscr{B}}$ in Eq. (12) into Eq. (20) we obtain

$$
\sigma \mathscr{P}_{12}=\mathscr{A} \mathscr{P}_{12} \hat{\mathscr{A}}^{\mathrm{T}}+\mathscr{B} \mathscr{B}{ }^{\mathrm{T}} \sigma \mathscr{L}^{\mathrm{T}} .
$$


Further, we substitute $\mathscr{B} \mathscr{B}^{\mathrm{T}}=\sigma \mathscr{S}^{\mathrm{T}} \sigma \mathscr{S}-\mathscr{A} \mathscr{S}^{\mathrm{T}} \mathscr{S} \mathscr{A}^{\mathrm{T}}$ and $\mathscr{L}=\widetilde{\Sigma}^{-1 / 2} \mathscr{U}_{1}^{\mathrm{T}} \mathscr{R}$ in the above equation and rearrange the terms to get

$$
\sigma\left(\mathscr{P}_{12}-\mathscr{S}^{\mathrm{T}} \mathscr{S} \mathscr{R}^{\mathrm{T}} \mathscr{U}_{1} \widetilde{\Sigma}^{-1 / 2}\right)=\mathscr{A} \mathscr{P}_{12} \widehat{\mathscr{A}}^{\mathrm{T}}-\mathscr{A} \mathscr{S}^{\mathrm{T}} \mathscr{S} \mathscr{A}^{\mathrm{T}} \sigma \mathscr{R}^{\mathrm{T}} \sigma \mathscr{U}_{1} \sigma \widetilde{\Sigma}^{-1 / 2}
$$

Using again the identity $\mathscr{V}_{1} \mathscr{V}_{1}^{\mathrm{T}}+\mathscr{V}_{2} \mathscr{V}_{2}^{\mathrm{T}}=I$ and Eq. (16), we get for the right-hand side of Eq. (21)

$$
\mathscr{A} \mathscr{P}_{12} \widehat{\mathscr{A}}^{\mathrm{T}}-\mathscr{A} \mathscr{S}^{\mathrm{T}}\left(\mathscr{V}_{1} \mathscr{V}_{1}^{\mathrm{T}}+\mathscr{V}_{2} \mathscr{V}_{2}^{\mathrm{T}}\right) \mathscr{S} \mathscr{A}^{\mathrm{T}} \sigma \mathscr{R}^{\mathrm{T}} \sigma \mathscr{U}_{1} \sigma \widetilde{\Sigma}^{-1 / 2}=\mathscr{A}\left(\mathscr{P}_{12}-\mathscr{S}^{\mathrm{T}} \mathscr{V}_{1} \widetilde{\Sigma}^{1 / 2}\right) \hat{\mathscr{A}}^{\mathrm{T}}
$$

where we also used the expression of $\widehat{\mathscr{A}}$ in Eq. (12). Finally, observing that

$$
\mathscr{S} \mathscr{R}^{\mathrm{T}} \mathscr{U}_{1}=\mathscr{V} \Sigma \mathscr{U}^{\mathrm{T}} \mathscr{U}_{1}=\mathscr{V}_{1} \widetilde{\Sigma}
$$

we get from Eq. (21)

$$
\sigma\left(\mathscr{P}_{12}-\mathscr{S}^{\mathrm{T}} \mathscr{V}_{1} \widetilde{\Sigma}^{1 / 2}\right)=\mathscr{A}\left(\mathscr{P}_{12}-\mathscr{S}^{\mathrm{T}} \mathscr{V}_{1} \widetilde{\Sigma}^{1 / 2}\right) \widehat{\mathscr{A}}^{\mathrm{T}}
$$

which has the unique solution

$$
\mathscr{P}_{12}=\mathscr{S}^{\mathrm{T}} \mathscr{V}_{1} \widetilde{\Sigma}^{1 / 2}
$$

Thus, the reachability Gramian is given by

$$
P_{k, e}=\left[\begin{array}{cc}
S_{k}^{\mathrm{T}} S_{k} & S_{k}^{\mathrm{T}} V_{k, 1} \widetilde{\Sigma}_{k}^{1 / 2} \\
\widetilde{\Sigma}_{k}^{1 / 2} V_{k, 1}^{\mathrm{T}} S_{k} & \widetilde{\Sigma}_{k}
\end{array}\right] .
$$

Similarly, we find that the observability Gramian is

$$
Q_{k, e}=\left[\begin{array}{cc}
R_{k}^{\mathrm{T}} R_{k} & -R_{k}^{\mathrm{T}} U_{k, 1} \widetilde{\Sigma}_{k}^{1 / 2} \\
-\widetilde{\Sigma}_{k}^{1 / 2} U_{k, 1}^{\mathrm{T}} R_{k} & \widetilde{\Sigma}_{k}
\end{array}\right] .
$$

A direct check shows that $\mathscr{P}_{e} \mathscr{Q}_{e}=0$ and therefore all Hankel singular values are zero. Using operator theory arguments [5], it results that the Hankel-operator associated to the error system is zero and thus the corresponding input-output (Toeplitz) operator is also zero. Thus the two systems realize the same input-output operator.

The computation of the minimal realization relies exclusively on square-root information (the Cholesky factors of Gramians) and this leads to a guaranteed enhancement of the overall numerical accuracy of computations. The key computation in determining the truncation matrices $\mathscr{L}$ and $\mathscr{T}$ is the solution of the two PDPLEs (6) and (7) with time-varying dimensions directly for the Cholesky factors of the Gramians. A numerically reliable procedure for this purpose is discussed in Section 5.

\section{Balancing-free square-root minimal realization}

To obtain a minimal realization from a non-minimal one we do not actually need to obtain a minimal realization which is balanced, since this could involve ill-conditioned truncation matrices $\mathscr{L}$ and $\mathscr{T}$, if the original system is poorly scaled. Since $\mathscr{L} \mathscr{T}=I$, the pair $(\mathscr{L}, \mathscr{T})$ defines a projector $\mathscr{T} \mathscr{L}$, in analogy to the 
case of standard systems. Thus, for any invertible $\mathscr{W}$, the pair of truncation matrices

$$
(\widetilde{\mathscr{L}}, \widetilde{\mathscr{T}})=\left(\mathscr{W}^{-1} \mathscr{L}, \mathscr{T} \mathscr{W}\right)
$$

defines the same projected system in a different coordinate form. Thus, to avoid potential accuracy losses, an alternative to balancing is to use a balancing-free approach to compute truncation matrices. In standard case, such a method has been proposed in [10]. A square-root balancing-free approach for the periodic case can be devised analogously as in case of standard systems [14], by combining the square-root method with a balancing-free method. Consider the QR-decompositions

$$
\mathscr{S}^{\mathrm{T}} \mathscr{V}_{1}=\widetilde{\mathscr{T}} \mathscr{X}, \quad \mathscr{R}^{\mathrm{T}} \mathscr{U}_{1}=\widetilde{\mathscr{Z}} \mathscr{Y},
$$

where $\mathscr{X}$ and $\mathscr{Y}$ are nonsingular matrices and $\widetilde{\mathscr{T}}$ and $\widetilde{\mathscr{Z}}$ are matrices with orthonormal columns. With the already computed $\widetilde{\mathscr{T}}$ we define the corresponding $\widetilde{\mathscr{L}}$ as

$$
\widetilde{\mathscr{L}}=\left(\widetilde{\mathscr{Z}}^{\mathrm{T}} \widetilde{\mathscr{T}}\right)^{-1} \widetilde{\mathscr{Z}}^{\mathrm{T}} \text {. }
$$

We have the following result analogous to Theorem 1 .

Theorem 2. The periodic system

$$
(\tilde{\mathscr{A}}, \widetilde{\mathscr{B}}, \widetilde{\mathscr{C}}):=(\sigma \widetilde{\mathscr{L}} \mathscr{A} \widetilde{\mathscr{T}}, \sigma \widetilde{\mathscr{L}} \mathscr{B}, \mathscr{C} \widetilde{\mathscr{T}})
$$

with $\widetilde{\mathscr{L}}$ and $\widetilde{\mathscr{T}}$ defined in Eqs. (22) and (23) is a minimal realization of the system $(\mathscr{A}, \mathscr{B}, \mathscr{C})$.

Proof. We show that the periodic system $(\widetilde{\mathscr{A}}, \widetilde{\mathscr{B}}, \widetilde{\mathscr{C}})$ is Lyapunov-similar to the balanced minimal realization (12). We observe that using Eqs. (22) and (23), we can relate the truncation matrices in Eq. (11) with $\widetilde{\mathscr{L}}$ and $\widetilde{T}$ as

$$
\mathscr{L}=\widetilde{\Sigma}^{-1 / 2} \mathscr{Y}^{\mathrm{T}} \widetilde{\mathscr{Z}}^{\mathrm{T}}=\widetilde{\Sigma}^{-1 / 2} \mathscr{Y}^{\mathrm{T}}\left(\widetilde{\mathscr{Z}}{ }^{\mathrm{T}} \widetilde{\mathscr{T}}\right) \widetilde{\mathscr{L}}, \quad \mathscr{T}=\widetilde{\mathscr{T}} \mathscr{X} \widetilde{\Sigma}^{-1 / 2} .
$$

To show that $(\widetilde{\mathscr{A}}, \widetilde{\mathscr{B}}, \widetilde{\mathscr{C}})$ and $(\widehat{\mathscr{A}}, \widehat{\mathscr{B}}, \widehat{\mathscr{C}})$ are Lyapunov-similar it is enough to show that

$$
\mathscr{W}=\widetilde{\Sigma}^{-1 / 2} \mathscr{Y}^{\mathrm{T}}\left(\widetilde{\mathscr{Z}}^{\mathrm{T}} \widetilde{\mathscr{T}}\right)
$$

is the corresponding balancing transformation having as its inverse $\mathscr{X} \widetilde{\Sigma}^{-1 / 2}$. But this follows by simply checking that

$$
\widetilde{\Sigma}^{-1 / 2} \mathscr{Y}^{\mathrm{T}}\left(\widetilde{\mathscr{Z}}^{\mathrm{T}} \widetilde{\mathscr{T}}\right) \mathscr{X} \widetilde{\Sigma}^{-1 / 2}=\widetilde{\Sigma}^{-1 / 2} \mathscr{U}_{1}^{\mathrm{T}} \mathscr{R} \mathscr{S}^{\mathrm{T}} \mathscr{V}_{1} \widetilde{\Sigma}^{-1 / 2}=I .
$$

Since the balancing-free square-root approach always constructs well-conditioned truncation matrices, the use of this method for poorly scaled systems leads usually to an improvement of the overall computational accuracy.

\section{Solution of PDPLEs}

The main computational problem to compute a balanced minimal realization of a periodic system consists in solving a pair of PDPLEs of the forms (6) and (7) directly for the Cholesky factor of the solutions. For the case of constant dimensions, algorithms to solve PDPLEs have been recently developed in [15]. These algorithms represent extensions of the method for standard systems proposed by Hammarling [7]. The new methods rely on an initial reduction of the periodic Lyapunov equations to simpler forms by using the periodic Schur decomposition of a product of square matrices [3]. The reduced equations are then solved by using 
special forward substitution algorithms. Important computational subproblems are in this context the efficient and numerically stable solution of order one or order two PDPLEs and of low order discrete periodic Sylvester equations. Computational approaches for these subproblems are also described in detail in [15].

In this section we describe an extension of the method of [15] to solve PDPLEs with time-varying dimensions. We only discuss the basic approach to solve the reverse-time PDPLE

$$
\mathscr{U}^{\mathrm{T}} \mathscr{U}=\mathscr{A}^{\mathrm{T}} \sigma \mathscr{U}^{\mathrm{T}} \sigma \mathscr{U} \mathscr{A}+\mathscr{R}^{\mathrm{T}} \mathscr{R}
$$

directly for the Cholesky factor $\mathscr{U}$, where $U_{k} \in \mathbb{R}^{n_{k} \times n_{k}}, A_{k} \in \mathbb{R}^{n_{k+1} \times n_{k}}, R_{k} \in \mathbb{R}^{n_{k} \times n_{k}}$, and the dimension $n_{k}$ are periodic with period $K \geqslant 1$. A completely analog approach can be derived for solving forward-time PDPLEs. To solve Eq. (24) we assume that for each $\tau$ the monodromy matrix $\Phi_{A}(\tau+K, \tau)$ has all characteristic multipliers in the open unit disk.

One class of methods to solve positive periodic Lyapunov equations can be devised along the lines of methods for periodic systems with constant state-space dimension [11]. This method is based on reducing these equations to a single standard positive discrete Lyapunov equation to compute a periodic generator, say $U_{0}$, which satisfies

$$
U_{0}^{\mathrm{T}} U_{0}=\Phi_{A}^{\mathrm{T}}(K, 0) U_{0}^{\mathrm{T}} U_{0} \Phi_{A}(K, 0)+\sum_{j=0}^{K-1} \Phi_{A}^{\mathrm{T}}(j, 0) R_{j}^{\mathrm{T}} R_{j} \Phi_{A}(j, 0) .
$$

Since $\Phi_{A}(K, 0) \in \mathbb{R}^{n_{0} \times n_{0}}$ is square, $U_{0} \in \mathbb{R}^{n_{0} \times n_{0}}$ can be computed by using Hammarling's method [7]. The rest of matrices $U_{k}, k=K-1, \ldots, 1$ results by recursion. Even with the enhancements similar to those proposed in [11], this approach is generally not recommendable because of implied matrix products and sums of matrix products. However, as it has been shown in [15], an iterative variant of this approach is well suited to solve PDPLEs, especially of low orders.

The periodic generator method allows one to justify a straightforward embedding of the problems with timevarying dimensions into larger order problems with constant dimension. Such an embedding allows us to solve the PDPLE (24) by using algorithms for constant dimension [15]. Let $\bar{n}=\max _{k}\left\{n_{k}\right\}$ and consider the extended $\bar{n} \times \bar{n} K$-periodic matrices

$$
A_{k, e}=\left[\begin{array}{cc}
A_{k} & 0 \\
0 & 0
\end{array}\right], \quad R_{k, e}=\left[\begin{array}{cc}
R_{k} & 0 \\
0 & 0
\end{array}\right], \quad U_{k, e}=\left[\begin{array}{cc}
U_{k} & 0 \\
0 & 0
\end{array}\right],
$$

where the zero matrices have appropriate dimensions. Then it is easy to see (for instance, by using the periodic generator approach) that $\mathscr{U}_{e}$ is the solution of the PDPLE

$$
\mathscr{U}_{e}^{\mathrm{T}} \mathscr{U}_{e}=\mathscr{A}_{e}^{\mathrm{T}} \sigma \mathscr{U}_{e}^{\mathrm{T}} \sigma \mathscr{U}_{e} \mathscr{A}_{e}+\mathscr{R}_{e}^{\mathrm{T}} \mathscr{R}_{e}
$$

with constant dimensions. The main drawback of this approach is that working with extended matrices with many zero elements leads to an unnecessary loss of computational efficiency. Alternatively, an efficient approach can be devised which fully exploits the underlying problem structure.

In the approach which we propose, we rely on an extension of the periodic Schur decomposition (PSD) of a cyclic product of square matrices and of the corresponding algorithms for its computation $[3,8]$.

Proposition 3. Let $A_{k} \in \mathbb{R}^{n_{k+1} \times n_{k}}, k=0,1, \ldots, K-1$, with $n_{K}=n_{0}$ be arbitrary matrices and let $\underline{n}=\min _{k}\left\{n_{k}\right\}$. Then there exist orthogonal matrices $Z_{k} \in \mathbb{R}^{n_{k} \times n_{k}}$ such that the matrices $\widetilde{A}_{k}=Z_{k+1}^{\mathrm{T}} A_{k} Z_{k}$ for $k=0, \ldots, k-1$ are block upper triangular

$$
\widetilde{A}_{k}=\left[\begin{array}{cc}
\widetilde{A}_{k, 11} & \widetilde{A}_{k, 12} \\
0 & \widetilde{A}_{k, 22}
\end{array}\right]
$$

where $\widetilde{A}_{k, 11} \in \mathbb{R}^{\underline{n} \times \underline{n}}, \widetilde{A}_{k, 22} \in \mathbb{R}^{\left(n_{k+1}-\underline{n}\right) \times\left(n_{k}-\underline{n}\right)}$ for $k=0,1, \ldots, K-1$. Moreover, $\widetilde{A}_{K-1,11}$ is in a real Schur form $(R S F), \widetilde{A}_{k, 11}$ for $k=0, \ldots, K-2$ are upper triangular and $\widetilde{A}_{k, 22}$ for $k=0, \ldots, K-1$ are upper trapezoidal. 
Proof. Let $p$ be such that $n_{p}=\underline{n}$ and without loss of generality we assume $p=0$. Let $U_{1} \in \mathbb{R}^{n_{1} \times n_{1}}$ be an orthogonal matrix such that

$$
U_{1}^{\mathrm{T}} A_{0}=\left[\begin{array}{c}
A_{0,11} \\
O
\end{array}\right],
$$

where $A_{0,11} \in \mathbb{R}^{\underline{n} \times \underline{n}}$. An upper triangular $A_{0,11}$ results for example from the $\mathrm{QR}$ decomposition of $A_{0}$. For $k=1, \ldots, K-1$, we successively determine the orthogonal matrices $U_{k+1} \in \mathbb{R}^{n_{k+1} \times n_{k+1}}$ such that each partitioned matrix

$$
U_{k+1}^{\mathrm{T}} A_{k} U_{k}=\left[\begin{array}{cc}
A_{k, 11} & A_{k, 12} \\
O & A_{k, 22}
\end{array}\right]
$$

is upper trapezoidal, $A_{k, 11} \in \mathbb{R}^{\underline{n} \times \underline{n}}$ and $A_{k, 22} \in \mathbb{R}^{\left(n_{k+1}-\underline{n}\right) \times\left(n_{k}-\underline{n}\right)}$. At this moment, we redefine $A_{0,11}$ from

$$
U_{1}^{\mathrm{T}} A_{0} U_{K-1}=\left[\begin{array}{c}
A_{0,11} \\
O
\end{array}\right] .
$$

Notice that, excepting the leading diagonal matrices, the transformed matrices $U_{k+1}^{\mathrm{T}} A_{k} U_{k}$ have the requested form in Eq. (28). To finish, we use the results of [3] to determine the orthogonal matrices $Q_{k} \in \mathbb{R} \underline{n} \times \underline{n}$ which bring the product $A_{K-1,11} \cdots A_{0,11}$ in RSF using the associated PSD. The overall transformation matrices $Z_{k}$, achieving the desired form in Eq. (28), are given by $Z_{k}=U_{k} \cdot \operatorname{diag}\left(Q_{k}, I\right)$.

Note that by using the extended PSD instead of the PSD of the product of extended matrices, a notable reduction of computational costs could arise if the difference $\bar{n}-\underline{n}$ is significant. It can be easily verified that each $\Phi_{\tilde{A}}(k+K, k)=Z_{k}^{\mathrm{T}} \Phi_{A}(k+K, k) Z_{k}$ is in a particular $\mathrm{RSF}$

$$
\Phi_{\tilde{A}}(k+K, k)=\left[\begin{array}{cc}
\Phi_{\tilde{A}_{11}}(k+K, k) & \times \\
0 & 0
\end{array}\right] .
$$

and each $\Lambda\left(\Phi_{\tilde{A}_{11}}(k+K, k)\right)$ defines the set of $\underline{n}$ core characteristic values (independent of $\left.k\right)$.

Let $\mathscr{Z}$ be an orthogonal Lyapunov transformation to compute the extended PSD of the monodromy matrix $\Phi_{A}(K, 0)$ in the Proposition 3 and define $\widetilde{\mathscr{A}}=\sigma \mathscr{Z}^{\mathrm{T}} \mathscr{A} \mathscr{Z}$ and the upper triangular $\widetilde{\mathscr{R}}$ such that $\widetilde{\mathscr{R}}^{\mathrm{T}} \widetilde{R}=\mathscr{Z}^{\mathrm{T}} \mathscr{R}^{\mathrm{T}} \mathscr{R} \mathscr{Z}$. Eq. (24) becomes after premultiplication with $\mathscr{Z}^{\mathrm{T}}$ and postmultiplication with $\mathscr{Z}$

$$
\widetilde{\mathscr{U}}^{\mathrm{T}} \widetilde{\mathscr{U}}=\tilde{\mathscr{A}}^{\mathrm{T}} \sigma \widetilde{\mathscr{U}}^{\mathrm{T}} \sigma \widetilde{\mathscr{U}} \tilde{\mathscr{A}}+\widetilde{\mathscr{R}}^{\mathrm{T}} \widetilde{\mathscr{R}},
$$

where $\widetilde{\mathscr{U}}=\mathscr{U} \mathscr{Z}$. After solving this reduced equation for $\widetilde{\mathscr{U}}$, the solution of Eq. (24) is obtained as $\mathscr{U}=\widetilde{\mathscr{U}} \mathscr{Z}^{\mathrm{T}}$. In order to simplify the notations, we assume in what follows that Eq. (24) is already in the reduced form (29) and $\mathscr{R}$ is upper triangular.

Let us partition $A_{k}, U_{k}$ and $R_{k}$ analogously as

$$
A_{k}=\left[\begin{array}{cc}
A_{k, 11} & A_{k, 12} \\
0 & A_{k, 22}
\end{array}\right], \quad U_{k}=\left[\begin{array}{cc}
U_{k, 11} & U_{k, 12} \\
0 & U_{k, 22}
\end{array}\right], \quad R_{k}=\left[\begin{array}{cc}
R_{k, 11} & R_{k, 12} \\
0 & R_{k, 22}
\end{array}\right],
$$

where the upper left blocks are $p_{1} \times p_{1}$ matrices $\left(p_{1}=1\right.$ or 2$)$. Assuming $\mathscr{U}_{11}$ non-singular we can derive recursive formulas which are analogous with those in the case of constant dimensions. By rewriting Eq. (24) for the partitioned matrices, we obtain

$$
\begin{aligned}
& \mathscr{U}_{11}^{\mathrm{T}} \mathscr{U}_{11}=\mathscr{A}_{11}^{\mathrm{T}} \sigma \mathscr{U}_{11}^{\mathrm{T}} \sigma \mathscr{U}_{11} \mathscr{A}_{11}+\mathscr{R}_{11}^{\mathrm{T}} \mathscr{R}_{11}, \\
& \mathscr{U}_{12}=\mathscr{M}_{1}^{\mathrm{T}} \sigma \mathscr{U}_{12} \mathscr{A}_{22}+\mathscr{M}_{1}^{\mathrm{T}} \sigma \mathscr{U}_{11} \mathscr{A}_{12}+\mathscr{M}_{2}^{\mathrm{T}} \mathscr{R}_{12}, \\
& \mathscr{U}_{22}^{\mathrm{T}} \mathscr{U}_{22}=\mathscr{A}_{22}^{\mathrm{T}} \sigma \mathscr{U}_{22}^{\mathrm{T}} \sigma \mathscr{U}_{22} \mathscr{A}_{22}+\mathscr{R}_{22}^{\mathrm{T}} \mathscr{R}_{22}+\mathscr{Y}^{\mathrm{T}} \mathscr{Y},
\end{aligned}
$$


where $\mathscr{M}_{1}=\sigma \mathscr{U}_{11} \mathscr{A}_{11} \mathscr{U}_{11}^{-1}, \mathscr{M}_{2}=\mathscr{R}_{11} \mathscr{U}_{11}^{-1}$, and $\mathscr{Y}$ is defined by the $K$-periodic matrix

$$
Y_{k}=N_{k}\left[\begin{array}{c}
R_{k, 12} \\
U_{k+1,11} A_{k, 12}+U_{k+1,12} A_{k, 22}
\end{array}\right]
$$

with each $N_{k}$ satisfying

$$
P_{k}=I_{2 p_{1}}-\left[\begin{array}{l}
M_{k, 2} \\
M_{k, 1}
\end{array}\right]\left[\begin{array}{l}
M_{k, 2} \\
M_{k, 1}
\end{array}\right]^{\mathrm{T}}=N_{k}^{\mathrm{T}} N_{k} .
$$

It can be shown that $P_{k}=P_{k}^{2} \geqslant 0$ and $\operatorname{rank} P_{k}=p_{1}$. Moreover, because

$$
P_{k}\left[\begin{array}{c}
M_{k, 2} \\
M_{k, 1}
\end{array}\right]=0
$$

$N_{k}$ can be computed as $N_{k}=Q_{k, 2}^{\mathrm{T}}$ from the QR-decomposition

$$
\left[\begin{array}{l}
M_{k, 2} \\
M_{k, 1}
\end{array}\right]=\left[Q_{k, 1} Q_{k, 2}\right]\left[\begin{array}{c}
V_{k} \\
0
\end{array}\right] .
$$

Thus by solving successively the first and second equation from Eq. (30) for $\mathscr{U}_{11}$ and $\mathscr{U}_{12}$, respectively, it remains to solve the third equation, where each $U_{k, 22}$ has lower order $n_{k}-p_{1}$. After updating the Cholesky factorization the resulting PDPLE for $\mathscr{U}_{22}$ has the same reduced form as the starting equation. The only difference between this procedure and that one in [15] for constant dimension is that after several steps some dimensions become zero. In such cases, the computations can continue without any breaks because, we can freely assume that the missing blocks in all matrices are zero matrices. For the rest, the algorithmic details are almost the same as in case of the procedure for constant dimension, although the efficient implementation for time-varying dimensions certainly requires an increased bookkeeping effort. For more details refer to [15].

Remark. To compute the Cholesky factors of the reachability and observability Gramians a single computation of the extended PSD of the monodromy matrix $\Phi_{A}(K, 0)$ is sufficient. In this way, the cost to solve the two PDPLEs (6) and (7) is almost the same as the cost of solving a single PDPLE.

\section{Conclusion}

We proposed a numerically sound approach to compute minimal realizations of linear periodic systems with time-varying dimensions. The proposed approach relies on algorithms using exclusively square-root information in form of Cholesky factors of the Gramians and therefore they have guaranteed enhanced computational accuracy. A square-root balancing-free variant has been derived, which we believe to be a completely satisfactory numerical approach to solve periodic minimal realization problems. The key computation in the proposed approach is the numerical solution of PDPLEs directly for the Cholesky factors of the solutions. A numerically reliable computational algorithm has been proposed to solve PDPLEs with varying dimension. This algorithm extends similar algorithms to solve PDPLEs with constant dimension.

The proposed minimal realization methods for periodic systems are not restricted to asymptotically stable periodic systems. For an unstable system, a simple scaling can be used to enforce the stability of the starting representation. For instance, it is possible to replace only $A_{0}$ by $\alpha A_{0}$, where $0<\alpha<1$ is chosen such that $\alpha \Phi_{A}(K, 0)$ has eigenvalues in the open unit disc. For the $\alpha$-scaled system, we can apply either the square-root or balancing-free square-root approach to determine a minimal system. Finally, the computed $\widehat{A}_{0}$ or $\widetilde{A}_{0}$ needs to be rescaled to $\widehat{A}_{0} / \alpha$ or $\widetilde{A}_{0} / \alpha$, respectively. Note that even in case of using the square-root approach on a $\alpha$-scaled system, the resulting rescaled minimal system is not balanced.

We expect that the proposed approach also provides a satisfactory computational solution to the balancing based model reduction of stable periodic systems. In this case, the truncation matrices have to be computed 
according to a separation of Hankel singular values with respect to a given tolerance, in analogy with the standard systems case. Note that even for constant dimension periodic systems, the resulting reduced models have in general time-varying dimensions, because, contrary to [12], generally we can not assume any interlacing properties for the singular values. This aspect seems to explain the lack of a complete theory of model reduction for periodic systems.

\section{References}

[1] S. Bittanti, P. Colaneri, Analysis of discrete-time linear periodic systems, in: C.T. Leondes (Ed.), Digital Control and Signal Processing Systems and Techniques, Control and Dynamics Systems, vol. 78, Academic Press, New York, 1996, pp. 313-339.

[2] S. Bittanti, P. Colaneri, G. De Nicolao, The difference periodic Riccati equation for the periodic prediction problem, IEEE Trans. Automat. Control 33 (1988) 706-712.

[3] A.W. Bojanczyk, G. Golub, P. Van Dooren, The periodic Schur decomposition, Algorithms and applications, in: F.T. Luk (Ed.), Proc. SPIE Conf., vol. 1770, 1992, pp. 31-42.

[4] P. Colaneri, S. Longhi, The realization problem for linear periodic systems, Automatica 31 (1995) 775-779.

[5] I. Gohberg, M.A. Kaashoek, L. Lerer, Minimality and realization of discrete time-varying systems, Operator Theory: Adv. Appl. 56 (1992) 261-296.

[6] O.M. Grasselli, S. Longhi, Pole-placement for nonreachable periodic discrete-time systems, Math. Control Signals Syst. 4 (1991) $439-455$.

[7] S.J. Hammarling, Numerical solution of the stable, non-negative definite Lyapunov equation, IMA J. Numer. Anal. 2 (1982) $303-323$.

[8] J.J. Hench, A.J. Laub, Numerical solution of the discrete-time periodic Riccati equation, IEEE Trans. Automat. Control 39 (1994) 1197-1210.

[9] S. Longhi, R. Zulli, A note on robust pole assignment for periodic systems, IEEE Trans. Automat. Control 41 (1996) $1493-1497$.

[10] M.G. Safonov, R.Y. Chiang, A Schur method for balanced-truncation model reduction, IEEE Trans. Automat. Control 34 (1989) 729-733.

[11] J. Sreedhar, P. Van Dooren, Periodic Schur form and some matrix equations, in: U. Helmke, R. Mennicken, J. Saurer (Eds.), Proc. MTNS'93, Regensburg, Germany, Academic Verlag, Berlin, vol. I, 1993, pp. 339-362.

[12] B. Xie, R.K.A.V. Aripirala, V. Syrmos, Model reduction of linear discrete-time periodic systems using Hankel-norm approximations, in: Proc. 13th IFAC Congress, San Francisco, USA, 1996, pp. 245-250.

[13] M.S. Tombs, I. Postlethwaite, Truncated balanced realization of a stable non-minimal state-space system, Int. J. Control 46 (1987) 1319-1330.

[14] A. Varga, Efficient minimal realization procedure based on balancing, in: A. El Moudni P. Borne, S.G. Tzafestas (Eds.), Prepr. of IMACS Symp. on Modelling and Control of Technological Systems, vol. 2, 1991, pp. 42-47.

[15] A. Varga, Periodic Lyapunov equations: some applications and new algorithms, Int. J. Control 67 (1997) 69-87.

[16] A. Varga, S. Pieters, Gradient-based approach to solve optimal periodic output feedback control problems, Automatica 34 (1998) $477-481$. 